\title{
Editor's Message to Special Issue on Embedded Systems Engineering
}

\author{
EIICHI HaYAKaWA ${ }^{1, \text { a) }}$
}

Embedded systems support our social life and industries as "brains" in a wide variety of electronic products such as automobile vehicles, home appliances, industrial equipment, and telecommunication devices. Research and development for these systems require innovative ideas to integrate overall technologies from hardware to software, from fundamentals to applications since embedded systems have to realize new services and features under severe physical and economic constraints. Establishing and advancing the embedded system engineering are crucial not only to develop better products but to keep competitiveness of industries. Although Japan allegedly has some strength in embedded system industry, overseas research activities for embedded systems, mainly in Europe and the U.S., have rapidly progressed, and a large number of outcomes have been published. It is critical vigorously to promote research in universities and research institutions in Japan.

In this special issue, we called for papers from industry and academia on a broad range of areas including hardware and software technologies, project managements, and human resources and educations related to embedded systems. Especially, we encouraged to submit papers presented in "Embedded System Symposium (ESS)," which is sponsored by Special Interesting Group on Embedded Systems in Information Processing Society of Japan.

The number of submitted paper is 17 and 4 excellent papers have been accepted. 16 reviewers assigned from the editorial committee shown below conducted peer-reviewing.

We should accelerate to establish embedded system technologies through collaboration between industry and educational institutions. Since the special issues activate the collaboration, we plan to continue publishing them twice a year.

We would like to express our sincere appreciation to all the authors for their contribution.

\author{
The Editorial Committee
}

- Editor in-Chief:

Eiichi Hayakawa (Takushoku University)

- Deputy Chief Editor:

Hironori Nakajo (Tokyo University of Agriculture and Technology)

Kiyofumi Tanaka (Japan Advanced Institute of Science and Technology)

- Editorial Committee:

Masato Edahiro (Nagoya University)

Kouichi Ono (IBM Research - Tokyo)

Kenji Kise (Tokyo Institute of Technology)

Takashi Kitamura (National Institute of Advanced Industrial

Science and Technology)

Yoshinori Takeuchi (Osaka University)

Takaaki Tateishi (IBM Research - Tokyo)

Kenji Tei (National Institute of Informatics)

Yukikazu Nakamoto (University of Hyogo)

Natsuko Noda (Shibaura Institute of Technology)

Mikio Hashimoto (Toshiba Corporation)

Hiroaki Fukuda (Shibaura Institute of Technology)

Hiroki Matsutani (Keio University)

Takanori Yokoyama (Tokyo City University)

Yasutaka Wada (Meisei University) 